\title{
Lung and drugs in rheumatoid arthritis
}

\section{Opinion}

Many respiratory manifestations have been observed in rheumatoid arthritis (RA), they can be specific of the disease, infectious or due to drugs. The rheumatoid lung represents one of the most frequent extra-articular manifestation of RA, it may concern the pleura $(15 \%)$ such as the lymphocytic pleurisy, aseptic empyema and the fibro thorax, the pulmonary parenchyma (pulmonary nodules $20 \%$, interstitial infiltrative pneumonia 20\%) and airways (bronchiolitis and bronchiectasis $30 \%$, follicular bronchiolitis, constructive bronchiolitis). These manifestations are often interlinked and can sometimes complicate the diagnostic investigation. They are subclinical in $50 \%$ of RA and are symptomatic only in $10 \%$. Great progress has been made in immunopathology and in tom densitometry High Resolution (TDMHR).

Treatments currently used in RA (sulfasalazine, methotrexate, leflunomide, anti $\mathrm{TNF} \alpha$, rituximab) are not sheltered from respiratory complications and most of them are pneumo-toxic. interstitial lung disease may be observed, especially with methotrexate and leflunomide, they require the discontinuation of these drugs, a corticosteroid therapy in the first case and a wash out with cholestyramine or active carbon in case of Leflunomid intolerance.

Methotrexate represents the reference treatment of RA, it is used alone or in combination with other drugs or biotherapy. Many pulmonary complications are related to methotrexate. Acute pneumopathy and subacute pneumopathy "of hypersensitivity" $(0,5-$ $7 \%$ ) is unpredictable, rare, independent of dose, of the duration and of route of administration of the treatment, fortunately corticosensible.

Risk factors are age over 60 years, diabetes, hypoalbuminemia, kidney failure, smoking, underlying respiratory disease and previous treatments with gold and D-penicillamine. Clinically, it is manifested as dyspnea, fever, dry cough, and crepitations. Biologically, moderate hypereosinophilia, X-rays shows an interstitial syndrome (TDM interest). Pulmonary function shows a restrictive lung disease. Bronchoalveolar lavage shows lymphocytic dry socket (CD8+). The differential diagnosis is mainly done with infectious pneumonia. The therapeutic management involves the discontinuation of

\author{
Volume 2 Issue 4 - 2015
}

\section{Chafia Dahou Makhloufi}

Department of Rheumatology, Bab El Oued Hospital,Algeria

Correspondence: Chafia Dahou Makhloufi, Department of Rheumatology, Bab El Oued Hospital, BD Said Touati, Algiers, Algeria, Tel 0021321960474,

Email makhloufi-dahou@hotmail.com

Received: April 07, 2015 | Published: April II, 2015

methotrexate, oxygen-therapy and corticostreroid therapy. Mortality rate is estimated at $15-17 \%$.

Other manifestations have been described in RA, such as opportunistic infections with intracellular germ: pneumocystis pneumonia, nocardiosis, aspergillosis, cytomegalovirus, tuberculosis and atypic mycobacteria. The risk of tuberculosis is higher for patients treated by anti-TNF $\alpha$, it is a major concern and that is why health authorities made effective recommendations. Screening for patients with risks, the systematic treatment of latent tuberculosis before anti $\mathrm{TNF} \boldsymbol{\alpha}$ therapy and rigorous oversight of patients undergoing therapy, reduce the risk of tuberculosis.

Finally, the majority of treatments used in RA may be pneumotoxic. The practitioner must be notified of this type of potential side effects. The diagnosis of lung damage related to drugs must not be held before discounting any cardiac damage, specific or infectious.

\section{Acknowledgements}

None.

\section{Conflict of interest}

The author declares no conflict of interest. 